\title{
BMC Gastroenterology reviewer acknowledgement 2014
}

Magdalena Morawska

\section{Contributing reviewers}

The editors of BMC Gastroenterology would like to thank all our reviewers who have contributed to the journal in Volume 14 (2014).

Sumadiono

Indonesia

Monica Acalovschi

Romania

Stefan Acosta

Sweden

Yuichi Adachi

Japan

Luigi Adinolfi

Italy

Silvia Affo

USA

Nishat Afroz

India

Vikas Agarwal

India

Rakesh Aggarwal

India

Ashish Aggarwal

USA

Alessio Aghemo

Italy

Nikhil Agrawal

India

Ferdinando Agresta

Italy

Siv Ahrne

Sweden

\author{
Guruprasad Aithal \\ UK \\ Holly Algood \\ USA
}

Alina Allen

USA

Piero Luigi Almasio

Italy

Amin Alousi

USA

Akif Altinbas

Turkey

Deepak Amarapurkar

India

Samad Amini-Bavil-Olyaee

USA

Javier Ampuero

Spain

Ashwin Ananthakrishnan

USA

Emma Anderson

UK

Pietro Andreone

Italy

Francesco Angelico

Italy

Paul Angulo

USA

\author{
Donald Anthony \\ USA \\ Kunihiko Aoyagi \\ Japan
}

Teoman Apan

Turkey

Tomio Arai

Japan

Joop Arends

Netherlands

Juan Armendariz-Borunda Mexico

Alessandro Armuzzi

Italy

Marcella Arru

Italy

David Assis

USA

Ajax Atta

Brazil

Phil Austin

UK

Ali Azarm

USA

Lukas Büngens

Germany

Yi Ba

China

Correspondence: Magdalena.Morawska@biomedcentral.com

BioMed Central, Floor 6, 236 Gray's Inn Road, London WC1X 8HB, UK 


\begin{tabular}{|c|c|c|}
\hline $\begin{array}{l}\text { Hideo Baba } \\
\text { Japan }\end{array}$ & $\begin{array}{l}\text { Simona Bertoni } \\
\text { Italy }\end{array}$ & $\begin{array}{l}\text { Anders Boyd } \\
\text { France }\end{array}$ \\
\hline $\begin{array}{l}\text { Zahra Babaei } \\
\text { Iran }\end{array}$ & $\begin{array}{l}\text { Mirko Bertozzi } \\
\text { Italy }\end{array}$ & $\begin{array}{l}\text { Patrizia Brigidi } \\
\text { Italy }\end{array}$ \\
\hline $\begin{array}{l}\text { Alfa Hsing Chen Bai } \\
\text { Hong Kong }\end{array}$ & $\begin{array}{l}\text { Annalisa Berzigotti } \\
\text { Spain }\end{array}$ & $\begin{array}{l}\text { Giuseppe Brisinda } \\
\text { Italy }\end{array}$ \\
\hline $\begin{array}{l}\text { Sarah Ballou } \\
\text { USA }\end{array}$ & $\begin{array}{l}\text { Emilie Bessède } \\
\text { France }\end{array}$ & $\begin{array}{l}\text { Aurore Brondex } \\
\text { France }\end{array}$ \\
\hline $\begin{array}{l}\text { Ji Young Bang } \\
\text { USA }\end{array}$ & $\begin{array}{l}\text { Jan Best } \\
\text { Germany }\end{array}$ & $\begin{array}{l}\text { Jan Bruensing } \\
\text { Germany }\end{array}$ \\
\hline $\begin{array}{l}\text { Bulent Baran } \\
\text { Turkey }\end{array}$ & $\begin{array}{l}\text { Mark Bettington } \\
\text { Australia }\end{array}$ & $\begin{array}{l}\text { Sylvia Brugman } \\
\text { Netherlands }\end{array}$ \\
\hline $\begin{array}{l}\text { Varenka J Barbero Becerra } \\
\text { Mexico }\end{array}$ & $\begin{array}{l}\text { Aneel Bhangu } \\
\text { UK }\end{array}$ & $\begin{array}{l}\text { Stanislas Bruley Des Varannes } \\
\text { France }\end{array}$ \\
\hline $\begin{array}{l}\text { Marisa Bare } \\
\text { Spain }\end{array}$ & $\begin{array}{l}\text { Bhushan Bhole } \\
\text { India }\end{array}$ & $\begin{array}{l}\text { Christine Budke } \\
\text { USA }\end{array}$ \\
\hline $\begin{array}{l}\text { Savio Barreto } \\
\text { India }\end{array}$ & $\begin{array}{l}\text { Federico Biagi } \\
\text { Italy }\end{array}$ & $\begin{array}{l}\text { Anthony Buisson } \\
\text { France }\end{array}$ \\
\hline $\begin{array}{l}\text { Anushka Baruah } \\
\text { USA }\end{array}$ & $\begin{array}{l}\text { Veerle Bieghs } \\
\text { Germany }\end{array}$ & $\begin{array}{l}\text { Christophe Burucoa } \\
\text { France }\end{array}$ \\
\hline $\begin{array}{l}\text { Guido Basilisco } \\
\text { Jamaica }\end{array}$ & $\begin{array}{l}\text { Alberto Biondi } \\
\text { Italy }\end{array}$ & $\begin{array}{l}\text { Peter Bytzer } \\
\text { Denmark }\end{array}$ \\
\hline $\begin{array}{l}\text { Daniela Basso } \\
\text { Italy }\end{array}$ & $\begin{array}{l}\text { Edward Bixler } \\
\text { USA }\end{array}$ & $\begin{array}{l}\text { Karel Caca } \\
\text { Germany }\end{array}$ \\
\hline $\begin{array}{l}\text { Joana Bastos } \\
\text { Portugal }\end{array}$ & $\begin{array}{l}\text { Wojciech Blonski } \\
\text { USA }\end{array}$ & $\begin{array}{l}\text { Sanjun Cai } \\
\text { China }\end{array}$ \\
\hline $\begin{array}{l}\text { Tom Bates } \\
\text { UK }\end{array}$ & $\begin{array}{l}\text { Anneloes Bohte } \\
\text { Netherlands }\end{array}$ & $\begin{array}{l}\text { Roberto Calcedo } \\
\text { USA }\end{array}$ \\
\hline $\begin{array}{l}\text { Ian Beales } \\
\text { UK }\end{array}$ & $\begin{array}{l}\text { Bernd Bokemeyer } \\
\text { Germany }\end{array}$ & $\begin{array}{l}\text { Vincenza Calvaruso } \\
\text { Italy }\end{array}$ \\
\hline $\begin{array}{l}\text { Andrew Beggs } \\
\text { UK }\end{array}$ & $\begin{array}{l}\text { Eduardo Bondan } \\
\text { Brazil }\end{array}$ & $\begin{array}{l}\text { M. Constanza Camargo } \\
\text { USA }\end{array}$ \\
\hline $\begin{array}{l}\text { Stefano Bellentani } \\
\text { Italy }\end{array}$ & $\begin{array}{l}\text { Ole Bonderup } \\
\text { Denmark }\end{array}$ & $\begin{array}{l}\text { Ali Canbay } \\
\text { Germany }\end{array}$ \\
\hline $\begin{array}{l}\text { Massimo Bellini } \\
\text { Italy }\end{array}$ & $\begin{array}{l}\text { Joel Bornstein } \\
\text { Australia }\end{array}$ & $\begin{array}{l}\text { Rocco Cappellesso } \\
\text { Italy }\end{array}$ \\
\hline $\begin{array}{l}\text { Amine Benkabbou } \\
\text { Morocco }\end{array}$ & $\begin{array}{l}\text { Linda Jw Bosch } \\
\text { Netherlands }\end{array}$ & $\begin{array}{l}\text { Maria Cappello } \\
\text { Italy }\end{array}$ \\
\hline $\begin{array}{l}\text { Tim Benning } \\
\text { Netherlands }\end{array}$ & $\begin{array}{l}\text { Xavier Bossuyt } \\
\text { Belgium }\end{array}$ & $\begin{array}{l}\text { Gabriele Capurso } \\
\text { Italy }\end{array}$ \\
\hline $\begin{array}{l}\text { Shay Ben-Shachar } \\
\text { Israel }\end{array}$ & $\begin{array}{l}\text { Alex Bottle } \\
\text { UK }\end{array}$ & $\begin{array}{l}\text { Paolo Caraceni } \\
\text { Italy }\end{array}$ \\
\hline $\begin{array}{l}\text { Charles Bernstein } \\
\text { Canada }\end{array}$ & $\begin{array}{l}\text { Christopher Bowlus } \\
\text { USA }\end{array}$ & $\begin{array}{l}\text { Andrea Cariati } \\
\text { Italy }\end{array}$ \\
\hline
\end{tabular}


Bertrand Cariou

France

Ignazio Castagliuolo

Italy

Renato Caviglia

Italy

Sang Hoon Cha

South Korea

Heyson Chan

Hong Kong

Walter Chan

USA

David Chang

UK

Christophe Chassard

Switzerland

Hans Chen

China

Yung-Tai Chen

Taiwan

Alfred Cheng

Hong Kong

Daye Cheng

China

Toshimi Chiba

Japan

Yi-Chun Chiu

Taiwan

Minsig Choi

USA

Jinah Choi

USA

Bruno Christ

Germany

Chen Shuan Chung

Taiwan

Marco Cicardi

Italy

Rachele Ciccocioppo

Italy

Francois X Claret

USA

Irene Coati

Italy
Jeremy Cobbold

UK

Lori Coburn

USA

Audrey Coilly

France

Daniel Compagno

Argentina

Jason Coombes

UK

Gareth Corbett

UK

Renato Costi

Italy

José Cotter

Portugal

Jason Craggs

USA

Timothy Craig

USA

Cesare Cremon

Italy

Antonino Crinò

Italy

Piotr Czauderna

Poland

Chia-Yen Dai

Taiwan

Heather Dawson

Switzerland

Nicola De Bortoli

Italy

Arjuna De Silva

Sri Lanka

Antonio De Vincentis

Italy

Thomas De Wijkerslooth

Netherlands

Chris Dekaney

USA

Ihsan Ekin Demir

Germany

Mark Deneau

Canada
Mohammad Derakhshan

UK

Wim Derave

Belgium

Renumathy Dhanasekaran

USA

Puneet Dhar

India

Francesco Di Mario

Italy

Massimiliano Di Pietro

UK

Antonio Di Sabatino

Italy

Christoph Dietrich

Germany

John Dillon

UK

Linda Dirven

Netherlands

Sanjay Dixit

UK

Massimo Dominici

Italy

Henry Dong

USA

Jia-Hong Dong

China

Paola Dongiovanni

Italy

Steven Dooley

Germany

Maria Pina Dore

Italy

Richard Douard

France

Alexander Dowli

USA

Peter Draganov

USA

Chelsea Drennan

USA

Caigan Du

Canada 
Pradeep Dudeja

USA

Dan Dumitrascu

Romania

Ximena Duque

Mexico

Dayna Early

USA

Mattias Ekstedt

Sweden

Hicham El Alaoui

France

Karima El Rhazi

Morocco

Abdul Elkadri

Canada

Magdy El-Salhy

Norway

Anne Line Engsbro

Denmark

Munechika Enjoji

Japan

Bang Wool Eom

South Korea

Rune Erichsen

Denmark

Mert Erkan

Germany

M Estep

USA

Antongiulio Faggiano

Italy

Ezio Falletto

Italy

Klaudia Farkas

Hungary

Giammarco Fava

Italy

Francesco Feo

Italy

Juan Manuel Fernández

Spain

Conrado M Fernandez Rodriguez Spain
Jose Fernandez-Cebrian

Spain

Davide Festi

Italy

Carmine Finelli

Italy

Mitchell Fink

USA

Gionata Fiorino

Italy

Marco P. Fisichella

USA

Ruggiero Francavilla

Italy

Francesco Franceschi

Italy

Luca Frulloni

Italy

Ezequiel Fuentes-Panana

Mexico

Takeshi Fujita

Japan

Manuele Furnari

Italy

Anthony Gamboa

USA

Carmelo Garcia-Monzon

Spain

Manuela Gariboldi

Italy

Alfredo Garofalo

Italy

Antonio Gasbarrini

Italy

Christoph Gasche

Austria

Jeremie Gautheron

Germany

Maria Gazouli

Greece

Karel Geboes

Belgium

Krisztina Gecse

Hungary
Lorenzo Gemignani

Italy

Sotirios Georgopoulos

Greece

Uday Ghoshal

India

Peter Gillett

UK

Saulius Girnius

USA

Gabriel Glockzin

Germany

Petra Anna Golovics

Hungary

Ana M Gomez Pedrero

Spain

Fernando Gomollón García

Spain

Yihong Gong

China

Stevan Gonzalez

USA

Jeroen Goos

Netherlands

Vinod Gopalan

Australia

Ilyssa Gordon

USA

Eriko Goto

Japan

Corinne Gower-Rousseau

France

Richa Goyal

India

Sergio Gradilone

USA

Peter Green

USA

Christian Grieser

Germany

Stefania Grimaudo

Italy

Axel Grothey

USA 
Frank Grünhage

Germany

Stefano Guandalini

USA

Shruti Guhasarkar

India

Arjun Gupta

USA

Prabodh Gupta

USA

Sebastian Haag

Germany

Yoshio Haga

Japan

Lutz Hamann

Germany

Amira Hamzaoui

Tunisia

Jimin Han

South Korea

Yuyan Han

USA

Kenichi Harada

Japan

Simon Hart

UK

Muhammad Hasan

USA

Ammar Hassan

USA

John Hayman

Australia

Sugata Hazra

USA

Tianlin He

China

Paul Henderson

UK

Fernando Herbella

Brazil

Ulf Herbers

Germany

Rolando Hernández-Muñoz

Mexico
Klaus Herrlinger

Germany

Kerstin Herzer

Germany

Ludwig Heuss

Switzerland

Susumu Hijioka

Japan

Ida Hilmi

Malaysia

Kaveh Hoda

USA

Iva Hojak

Croatia

James Hollis

USA

Masaki Honda

Japan

Sam Hong

USA

Charlotte Hor Henrichsen

Spain

Kazutoshi Hori

Japan

Akira Horiuchi

Japan

Jan-Sing Hsieh

Taiwan

Jian $\mathrm{Hu}$

Hong Kong

Chun-Chieh Huang

Taiwan

Wolfgang Huber

Germany

Patrick Hughes

Australia

Ivan Fn Hung

Hong Kong

Niall Patrick Hyland

Ireland

Woo Jin Hyung

South Korea

Enzo Ierardi

Italy
Joep Ijspeert

Netherlands

Akira Imoto

Japan

Osamu Inatomi

Japan

Giuseppe Ingravallo

Italy

Pasquale Innominato

France

Paola Iovino

Italy

Hirotoshi Ishiwatari

Japan

Norio Isoda

Japan

Robert Issenman

Canada

Takao Itoi

Japan

Hrvoje Ivekovic

Croatia

Hartmut Jaeschke

USA

Anahita Jalilvand

USA

Lina Jansen

Germany

Thierry Jarde

Australia

Johannes Jeekel

Netherlands Antilles

Christian

Jenssen Germany

Hui Jiang

UK

Acer Jiang

China

Sabita Jiwnani

India

Lloris Jm

Spain

Nicolas Jonckheere

France 


\begin{tabular}{|c|c|c|}
\hline Michael Jones & Laurie Keefer & Vani Konda \\
\hline Australia & USA & USA \\
\hline Bharat Joshi & Simon Keely & Hanne Konradsen \\
\hline USA & Australia & Denmark \\
\hline Hwoon-Yong Jung & Daniel Keszthelyi & Christos Kontos \\
\hline South Korea & Netherlands & Greece \\
\hline Ali Kabir & Shara Ket & Marcela Kopacova \\
\hline Iran & UK & Czech Republic \\
\hline Tatehiro Kagawa & Martin Keuchel & Uri Kopylov \\
\hline Japan & Germany & Canada \\
\hline Alisan Kahraman & Muhammad Khurram & Uri Kopylov \\
\hline Germany & Pakistan & Israel \\
\hline Marko Kalliomäki & Gwang Ha Kim & John Koskinas \\
\hline Finland & South Korea & Greece \\
\hline Sunjeev Kamboj & Jae Kim & Aletta Kraneveld \\
\hline UK & South Korea & Netherlands \\
\hline Terumi Kamisawa & Seung Up Kim & Wolfgang Kratzer \\
\hline Japan & South Korea & Germany \\
\hline Nobuo Kanai & Baek-Hui Kim & Andi Krumbholz \\
\hline Japan & South Korea & Germany \\
\hline Tatsuo Kanda & Lajos Kiss & Anja Kühl \\
\hline Japan & Hungary & Germany \\
\hline Umadevi Kandasamy & Masayuki Kitano & Roland Kuiper \\
\hline China & Japan & Netherlands \\
\hline Hyoun Woo Kang & Janis Klovins & Seray Külcü Çakmak \\
\hline South Korea & Latvia & Turkey \\
\hline Lakshmi Kannan & Johannes Kluwe & Hemant Kulkarni \\
\hline USA & Germany & USA \\
\hline Konstantinos Kantartzis & Mate Knabe & Teru Kumagi \\
\hline Germany & Germany & Japan \\
\hline Sung-Shuo Kao & C M Frank Kneepkens & Manoj Kumar \\
\hline Taiwan & Netherlands & India \\
\hline Paulo Kassab & Hideki Kobara & Juozas Kupcinskas \\
\hline Brazil & Japan & Lithuania \\
\hline Shingo Kato & Taku Kobayashi & Aysegul Kuskucu \\
\hline Japan & Japan & Turkey \\
\hline Konstantinos Katsanos & Oliver Owen Koch & Vincenzo La Mura \\
\hline Greece & Austria & Italy \\
\hline Seymour Katz & Rakesh Kochhar & Conor Lahiff \\
\hline USA & India & Ireland \\
\hline Gurjeet Kaur & Motohiro Kojima & Ming-Wei Lai \\
\hline Malaysia & Japan & Taiwan \\
\hline Pawel Kawalec & Kaija-Leena Kolho & Terry Lairmore \\
\hline Poland & Finland & USA \\
\hline
\end{tabular}


Peter Lakatos

Hungary

Brian Lam

USA

Kristina Lamas

Sweden

Malinee Laopaiboon

Thailand

Ryan Law

USA

Cedric Le May

France

Sang-Jeon Lee

South Korea

Yeong Yeh Lee

Malaysia

Philippe Lehours

France

Michael Leitzmann

USA

Marcis Leja

Latvia

Cosmas Rinaldi Lesmana

Indonesia

William Letson

USA

Barrett Levesque

USA

Philippe Lévy

France

James Lewis

USA

Ding-You Li

USA

Aiwu Li

China

Chuang-Chi Liaw

Taiwan

Jingmei Lin

USA

Hans Lin

Taiwan

Marcelo Linhares

Brazil
Chia-Yuan Liu

Taiwan

Gin-Ho Lo

Taiwan

Amedeo Lonardo

Italy

Dayami Lopez

USA

Albert Lowenfels

USA

$M$ Isabel Lucena

Spain

Claudio Luchini

Italy

Alexander Lukasz

Germany

Tao Luo

China

Holger Lutz

Germany

Chang-Sheng Ma

China

Benjamin Maasoumy

Germany

Mariana Machado

Portugal

Lloyd Mack

Canada

Subha Madhavan

USA

Mohammad Madhoun

USA

K S Madhusudhan

India

Mariantonia Maglio

Italy

Sanjiv Mahadeva

Malaysia

Govind Makharia

India

Atul Malhotra

Australia

Shyamapada Mandal

India
Hendrik Manner

Germany

Spilios Manolakopoulos

Greece

Aiping Mao

USA

Séverine Margeridon

France

Athanasios Marinis

Greece

Daniele Marrelli

Italy

Javier Martin

Spain

Allyson

Martinez USA

Luca

Mastracci Italy

Daiuske Masuda

Japan

Ryota Masuzaki

USA

Takahisa Matsuda

Japan

Hanno Matthaei

Germany

Maria Cristina Mazzilli

Italy

Andrew Mckay

Canada

Colin Mckay

UK

Tobias Meister

Germany

Anders Meller Donatsky

Denmark

Joshua Melson

USA

Nahum Méndez-Sánchez

Mexico

Fabrice Menegaux

France

Shahin Merat

Iran 


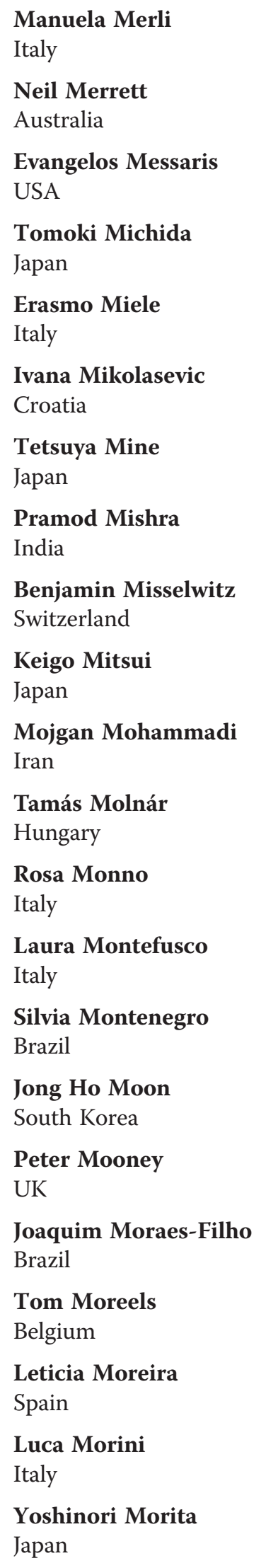

\author{
Chigusa Morizane \\ Japan \\ Mahmoud Mosli \\ Canada \\ Beat Peter Müller-Stich \\ Germany \\ Malaisamy Muniyandi \\ India
}

Atsuhiko Murata

Japan

Puja Myles

UK

Anna Myléus

Sweden

Alessio Naccarati

Italy

Takeaki Nagamine

Japan

Iris Nagtegaal

Netherlands

Shan Naidu

USA

Hayato Nakagawa

Japan

Shigemi Nakajima

Japan

Tadashi Nakamura

Japan

Udayakumar Navaneethan

USA

Rick Nelson

UK

Alessandro Neri

Italy

Yulia Nevzorova

Germany

Siew Ng

Hong Kong

Felix Nickel

Germany

Scott Nightingale

Australia

Mehrdad Nikfarjam

Australia
Akiyoshi Nishio

Japan

Mark Noar

USA

Camilla Nøjgaard

Denmark

Kristina Norman

Germany

Alireza Norouzi

Iran

Fredrik Norstrom

Sweden

Oscar Núñez Martínez

Spain

Sylvester Chuks Nwokediuko

Nigeria

Katsutoshi Obara

Japan

Stefan Oehlers

USA

Bodil Ohlsson

Sweden

Yuki Ohya

USA

Shiro Oka

Japan

Abdulfatai Olokoba

Nigeria

Ehinmidu Olorunmola Joseph Nigeria

Colm O'Morain

Ireland

Nikolaj Worm Orntoft

Denmark

Cécile Oury

Belgium

Nilufer Ozaydin

Turkey

Fabio Pace

Italy

Ganesh Pai

India

Sujoy Pal

India 


\begin{tabular}{|c|c|c|}
\hline $\begin{array}{l}\text { Grazia Palomba } \\
\text { Italy }\end{array}$ & $\begin{array}{l}\text { Oreste Pieramico } \\
\text { Italy }\end{array}$ & $\begin{array}{l}\text { Hari Ramesh } \\
\text { India }\end{array}$ \\
\hline $\begin{array}{l}\text { Anand Pandey } \\
\text { India }\end{array}$ & $\begin{array}{l}\text { Hubert Piessevaux } \\
\text { Belgium }\end{array}$ & $\begin{array}{l}\text { S.V. Rana } \\
\text { India }\end{array}$ \\
\hline $\begin{array}{l}\text { Mária Papp } \\
\text { Hungary }\end{array}$ & $\begin{array}{l}\text { Alessio Pini Prato } \\
\text { Italy }\end{array}$ & $\begin{array}{l}\text { Bettina M. Rau } \\
\text { Germany }\end{array}$ \\
\hline $\begin{array}{l}\text { Alfonso Papparella } \\
\text { Italy }\end{array}$ & $\begin{array}{l}\text { Alejandro Piscoya } \\
\text { Peru }\end{array}$ & $\begin{array}{l}\text { Debolina Ray } \\
\text { USA }\end{array}$ \\
\hline $\begin{array}{l}\text { Barbara Pardini } \\
\text { Czech Republic }\end{array}$ & $\begin{array}{l}\text { Marco Pizzi } \\
\text { Italy }\end{array}$ & $\begin{array}{l}\text { Kevin Reavis } \\
\text { USA }\end{array}$ \\
\hline $\begin{array}{l}\text { Se Jin Park } \\
\text { South Korea }\end{array}$ & $\begin{array}{l}\text { Philippe Poirier } \\
\text { France }\end{array}$ & $\begin{array}{l}\text { Helen Reeves } \\
\text { UK }\end{array}$ \\
\hline $\begin{array}{l}\text { Kt Park } \\
\text { USA }\end{array}$ & $\begin{array}{l}\text { Stefano Pontone } \\
\text { Italy }\end{array}$ & $\begin{array}{l}\text { Petr Ricanek } \\
\text { Norway }\end{array}$ \\
\hline $\begin{array}{l}\text { Rosa Maria Pascale } \\
\text { Italy }\end{array}$ & $\begin{array}{l}\text { Brian Poole } \\
\text { USA }\end{array}$ & $\begin{array}{l}\text { Claudio Ricci } \\
\text { Italy }\end{array}$ \\
\hline $\begin{array}{l}\text { Devis Pascut } \\
\text { Italy }\end{array}$ & $\begin{array}{l}\text { Mahmoudreza Pourkarim } \\
\text { Belgium }\end{array}$ & $\begin{array}{l}\text { Cristobal Richart } \\
\text { Spain }\end{array}$ \\
\hline $\begin{array}{l}\text { Michael Pavlides } \\
\text { UK }\end{array}$ & $\begin{array}{l}\text { Akram Pourshams } \\
\text { Iran }\end{array}$ & $\begin{array}{l}\text { Mark Riddle } \\
\text { USA }\end{array}$ \\
\hline $\begin{array}{l}\text { Anna Pecorelli } \\
\text { Italy }\end{array}$ & $\begin{array}{l}\text { C Pramesh } \\
\text { India }\end{array}$ & $\begin{array}{l}\text { Rachael Rigby } \\
\text { UK }\end{array}$ \\
\hline $\begin{array}{l}\text { George Peppas } \\
\text { Greece }\end{array}$ & $\begin{array}{l}\text { Rashmi Prasad } \\
\text { Sweden }\end{array}$ & $\begin{array}{l}\text { Luciana Rigoli } \\
\text { Italy }\end{array}$ \\
\hline $\begin{array}{l}\text { Montserrat Perez Encinas } \\
\text { Spain }\end{array}$ & $\begin{array}{l}\text { Timothy Price } \\
\text { Australia }\end{array}$ & $\begin{array}{l}\text { Cosmeri Rizzato } \\
\text { Italy }\end{array}$ \\
\hline $\begin{array}{l}\text { Angeles Perez-Aisa } \\
\text { Spain }\end{array}$ & $\begin{array}{l}\text { Lani Prideaux } \\
\text { Australia }\end{array}$ & $\begin{array}{l}\text { Barbera Roberta } \\
\text { Italy }\end{array}$ \\
\hline $\begin{array}{l}\text { Manuel Perez-Miranda } \\
\text { Spain }\end{array}$ & $\begin{array}{l}\text { Carlo Pulitano } \\
\text { Italy }\end{array}$ & $\begin{array}{l}\text { Martin Roderfeld } \\
\text { Germany }\end{array}$ \\
\hline $\begin{array}{l}\text { Giorgio Perilongo } \\
\text { Italy }\end{array}$ & $\begin{array}{l}\text { Giacomo Puppa } \\
\text { Italy }\end{array}$ & $\begin{array}{l}\text { Risto Roine } \\
\text { Finland }\end{array}$ \\
\hline $\begin{array}{l}\text { Jonathan Peterson } \\
\text { USA }\end{array}$ & $\begin{array}{l}\text { Katrina Purcell } \\
\text { Australia }\end{array}$ & $\begin{array}{l}\text { Anders Rönnblom } \\
\text { Sweden }\end{array}$ \\
\hline $\begin{array}{l}\text { Salvatore Petta } \\
\text { Italy }\end{array}$ & $\begin{array}{l}\text { Jorge Quiroga } \\
\text { Spain }\end{array}$ & $\begin{array}{l}\text { Kathleen Ross } \\
\text { UK }\end{array}$ \\
\hline $\begin{array}{l}\text { Raffaele Pezzilli } \\
\text { Italy }\end{array}$ & $\begin{array}{l}\text { István Rácz } \\
\text { Hungary }\end{array}$ & $\begin{array}{l}\text { Thomas Rossi } \\
\text { USA }\end{array}$ \\
\hline $\begin{array}{l}\text { Marco Picardi } \\
\text { Italy }\end{array}$ & $\begin{array}{l}\text { Robert Raffaniello } \\
\text { USA }\end{array}$ & $\begin{array}{l}\text { Christophe Rosty } \\
\text { Australia }\end{array}$ \\
\hline $\begin{array}{l}\text { Mathieu Piche } \\
\text { Canada }\end{array}$ & $\begin{array}{l}\text { Jayadev Raju } \\
\text { Canada }\end{array}$ & $\begin{array}{l}\text { Constance Ruhl } \\
\text { USA }\end{array}$ \\
\hline $\begin{array}{l}\text { Mercedes Pico } \\
\text { Argentina }\end{array}$ & $\begin{array}{l}\text { H Ramesh } \\
\text { India }\end{array}$ & $\begin{array}{l}\text { Mariagrazia Rumi } \\
\text { Italy }\end{array}$ \\
\hline
\end{tabular}




\begin{tabular}{|c|c|c|}
\hline Kimberly Ruscher & Gernot Sellge & Satu Simell \\
\hline USA & Germany & Finland \\
\hline Andrew Ruszkiewicz & Lawrence Serfaty & Harkirat Singh \\
\hline Australia & France & USA \\
\hline Stephen Ryder & Dionyssios Sgouras & Preet Paul Singh \\
\hline UK & Greece & USA \\
\hline Sammy Saab & Chirag N Shah & Rajvinder Singh \\
\hline USA & India & Australia \\
\hline Jayesh Sagar & Omar Shah & Shailender Singh \\
\hline UK & India & USA \\
\hline David Sahli & Tilak Shah & Siddharth Singh \\
\hline Sweden & USA & USA \\
\hline Yutaka Saito & Dea Shahinas & Iradj Sobhani \\
\hline Japan & Canada & France \\
\hline Anne Salonen & Ala Sharara & Ajit Sood \\
\hline Finland & Lebanon & India \\
\hline John Saltzman & Anamay Sharma & John Souglakos \\
\hline USA & USA & Greece \\
\hline Sundeep Saluja & Runhua Shi & Bridget Southwell \\
\hline India & USA & Australia \\
\hline Dorit Samocha-Bonet & Yuhong Shi & Valdenia Souza \\
\hline Australia & USA & Brazil \\
\hline Andres Sanchez-Yague & Yu-Lueng Shih & Donat Rudolf Spahn \\
\hline Spain & Taiwan & Switzerland \\
\hline Olof Sandström & Takaya Shimura & Marcel Spanier \\
\hline Sweden & Japan & Netherlands \\
\hline Fuat Saner & Cheol Min Shin & Ulrich Spengler \\
\hline Germany & South Korea & Germany \\
\hline Abbi Saniabadi & Yasuhiro Shirakawa & Robin Spiller \\
\hline Japan & Japan & UK \\
\hline Roberto Santambrogio & Elham Shirvani Dastgerdi & Ioan Sporea \\
\hline Italy & Germany & Romania \\
\hline Aaron Sasson & Ivy Shiue & Shanthi Srinivasan \\
\hline USA & UK & USA \\
\hline Edoardo Savarino & Yehuda Shoenfeld & Peter Stanich \\
\hline Italy & Israel & USA \\
\hline Stefano Scaringi & Shailesh Shrikhande & Robert Steele \\
\hline Italy & India & UK \\
\hline Carmelo Scarpignato & Nagini Siddavaram & Claudia Stefanutti \\
\hline Italy & India & Italy \\
\hline Michael Schumann & Parames Sil & Juergen Stein \\
\hline Germany & India & Germany \\
\hline Andrada Seicean & Michele Simbolo & Jayson Stoffman \\
\hline Romania & Italy & Canada \\
\hline
\end{tabular}


Jesse Stondell

USA

Kirsten Stone

USA

Antoinette Stroup

USA

Mitsushige Sugimoto

Japan

Ki Tae Suk

South Korea

Marianny Sulbaran

Brazil

Junfeng Sun

China

Xinchen Sun

China

Michael Surette

Canada

Seth Sweetser

USA

Lonnie Swift

UK

Jeffrey Swigris

USA

Ari Syam

Indonesia

James Tabibian

USA

Frank Tacke

Germany

Wei-Chen Tai

Taiwan

Diana Tait

UK

Toru Takahashi

Japan

Nagio Takigawa

Japan

Rupjyoti Talukdar

India

Gianluca Tamagno

Ireland

Naoto Tamai

Japan
Kevin Shyong-Wei Tan

Singapore

Manu Tandan

India

Raymond Tang

Hong Kong

Whitney Tang

Hong Kong

Marcel Tantau

Romania

Sudeep Tanwar

UK

Luc Tappy

Switzerland

Deniz Tastemir Korkmaz

Turkey

Ryosuke Tateishi

Japan

Felix Tellez-Avila

Mexico

Richard Ten Broek

Netherlands

Christopher Teshima

Canada

Jason Theis

USA

Gregory Thomas

UK

Chengju Tian

USA

Kim Timmermans

Netherlands

Dina Tiniakos

Greece

Jens Tischendorf

Germany

Liviu Titu

UK

Ervin Toth

Sweden

Takashi Toyonaga

Japan

Jonel Trebicka

Germany
William J. Trickler

USA

Andrea Tringali

Italy

Emmanuel Tsochatzis

UK

Emmanuel Tsochatzis

Greece

Toshio Tsuyuguchi

Japan

Tao-Hsin Tung

Taiwan

Nigel Turner

Australia

Antonio Tursi

Italy

Koji Uchino

Japan

Noriya Uedo

Japan

Hirofumi Uto

Japan

Francesco Valitutti

Italy

Mark Van Baal

Netherlands

David Van Der Poorten

Australia

Sascha Van Doorn

Netherlands

Tom Van Wezel

Netherlands

Sina Vatandoust

Australia

Maria Velasco

Spain

Sander Veldhuyzen Van Zanten

Canada

Julien Vergniol

France

Piero Vernia

Italy

Umberto Vespasiani-Gentilucci

Italy 


\begin{tabular}{|c|c|c|}
\hline $\begin{array}{l}\text { Michael Vieth } \\
\text { Germany }\end{array}$ & $\begin{array}{l}\text { Martin-Walter Welker } \\
\text { Germany }\end{array}$ & $\begin{array}{l}\text { Wancai Yang } \\
\text { China }\end{array}$ \\
\hline $\begin{array}{l}\text { Mauro Vigan\&Ograve } \\
\text { Italy }\end{array}$ & $\begin{array}{l}\text { John Windsor } \\
\text { New Zealand }\end{array}$ & $\begin{array}{l}\text { Kazuhiro Yasuda } \\
\text { Japan }\end{array}$ \\
\hline $\begin{array}{l}\text { Cristiane Villela-Nogueira } \\
\text { Brazil }\end{array}$ & $\begin{array}{l}\text { Tibor Wittmann } \\
\text { Hungary }\end{array}$ & $\begin{array}{l}\text { Burcu Yavuz } \\
\text { Turkey }\end{array}$ \\
\hline $\begin{array}{l}\text { Julio Villena } \\
\text { Argentina }\end{array}$ & $\begin{array}{l}\text { Sunny Hei Wong } \\
\text { Hong Kong }\end{array}$ & $\begin{array}{l}\text { Raymond Yeung } \\
\text { USA }\end{array}$ \\
\hline $\begin{array}{l}\text { Manlio Vinciguerra } \\
\text { UK }\end{array}$ & $\begin{array}{l}\text { Vincent Wong } \\
\text { Hong Kong }\end{array}$ & $\begin{array}{l}\text { Jung-Hwan Yoon South } \\
\text { Korea }\end{array}$ \\
\hline $\begin{array}{l}\text { Aron Vincze } \\
\text { Hungary }\end{array}$ & $\begin{array}{l}\text { Jeremy Woodward } \\
\text { UK }\end{array}$ & $\begin{array}{l}\text { Shuhei Yoshida } \\
\text { Japan }\end{array}$ \\
\hline $\begin{array}{l}\text { Jiannis Vlachogiannakos } \\
\text { Greece }\end{array}$ & $\begin{array}{l}\text { Marcus-Alexander Wörns } \\
\text { Germany }\end{array}$ & $\begin{array}{l}\text { Zili You } \\
\text { China }\end{array}$ \\
\hline $\begin{array}{l}\text { Umberto Volta } \\
\text { Italy }\end{array}$ & $\begin{array}{l}\text { Alexander Wree } \\
\text { USA }\end{array}$ & $\begin{array}{l}\text { Muhammad Zubair Yousaf } \\
\text { Pakistan }\end{array}$ \\
\hline $\begin{array}{l}\text { Atte Von Wright } \\
\text { Finland }\end{array}$ & $\begin{array}{l}\text { Han-Ping Wu } \\
\text { Taiwan }\end{array}$ & $\begin{array}{l}\text { Dominic Yu } \\
\text { UK }\end{array}$ \\
\hline $\begin{array}{l}\text { Ulrikke Voss } \\
\text { Sweden }\end{array}$ & $\begin{array}{l}\text { Deng-Chyang Wu } \\
\text { Taiwan }\end{array}$ & $\begin{array}{l}\text { Onchee Yu } \\
\text { USA }\end{array}$ \\
\hline $\begin{array}{l}\text { Amanda Waddell } \\
\text { USA }\end{array}$ & $\begin{array}{l}\text { Ming-Shiang Wu } \\
\text { Taiwan }\end{array}$ & $\begin{array}{l}\text { Haiyan Zhai } \\
\text { USA }\end{array}$ \\
\hline $\begin{array}{l}\text { Yasir Waheed } \\
\text { Pakistan }\end{array}$ & $\begin{array}{l}\text { Gabriele Wurm Johansson } \\
\text { Sweden }\end{array}$ & $\begin{array}{l}\text { Xingqi Zhang } \\
\text { China }\end{array}$ \\
\hline $\begin{array}{l}\text { Homan Wai } \\
\text { USA }\end{array}$ & $\begin{array}{l}\text { Xinhua Xiao } \\
\text { China }\end{array}$ & $\begin{array}{l}\text { Lin Zhang } \\
\text { China }\end{array}$ \\
\hline $\begin{array}{l}\text { Daniel Wall } \\
\text { UK }\end{array}$ & $\begin{array}{l}\text { Guifang Xu } \\
\text { China }\end{array}$ & $\begin{array}{l}\text { Qingchuan Zhao } \\
\text { China }\end{array}$ \\
\hline $\begin{array}{l}\text { David Wang } \\
\text { USA }\end{array}$ & $\begin{array}{l}\text { Shintaro Yagi } \\
\text { Japan }\end{array}$ & $\begin{array}{l}\text { Alexandra Zhernakova } \\
\text { Netherlands }\end{array}$ \\
\hline $\begin{array}{l}\text { Fusheng Wang } \\
\text { China }\end{array}$ & $\begin{array}{l}\text { Kazuhito Yajima } \\
\text { Japan }\end{array}$ & $\begin{array}{l}\text { Yan Zhong } \\
\text { China }\end{array}$ \\
\hline $\begin{array}{l}\text { Jin-Town Wang } \\
\text { Taiwan }\end{array}$ & $\begin{array}{l}\text { Javed Yakoob } \\
\text { Pakistan }\end{array}$ & $\begin{array}{l}\text { Henning Wolfgang Zimmermann } \\
\text { Germany }\end{array}$ \\
\hline $\begin{array}{l}\text { Hua Wang } \\
\text { China }\end{array}$ & $\begin{array}{l}\text { Sohsuke Yamada } \\
\text { Japan }\end{array}$ & $\begin{array}{l}\text { Alexander Zipprich } \\
\text { Germany }\end{array}$ \\
\hline $\begin{array}{l}\text { Mamoru Watanabe } \\
\text { Japan }\end{array}$ & $\begin{array}{l}\text { Yutaka Yamaji } \\
\text { Japan }\end{array}$ & $\begin{array}{l}\text { Duowu Zou } \\
\text { China }\end{array}$ \\
\hline $\begin{array}{l}\text { Ketut Dewi Kumara Wati } \\
\text { Indonesia }\end{array}$ & $\begin{array}{l}\text { Hiroki Yamaue } \\
\text { Japan }\end{array}$ & $\begin{array}{l}\text { Zhixiang Zuo } \\
\text { USA }\end{array}$ \\
\hline $\begin{array}{l}\text { Rabindra Watson } \\
\text { USA }\end{array}$ & $\begin{array}{l}\text { Kun Yan } \\
\text { China }\end{array}$ & \\
\hline $\begin{array}{l}\text { Jacques Weill } \\
\text { France }\end{array}$ & $\begin{array}{l}\text { Hwai-I Yang } \\
\text { Taiwan }\end{array}$ & \\
\hline
\end{tabular}

